\title{
ORIGINAL ARTICLE Vertical inhibition of the PI3K/Akt/mTOR pathway is synergistic in breast cancer
}

\author{
S-U Woo ${ }^{1,4}$, T Sangai ${ }^{1,5}$, A Akcakanat ${ }^{2}, \mathrm{H} \mathrm{Chen}^{3}, \mathrm{C} \mathrm{Wei}^{3,6}$ and F Meric-Bernstam ${ }^{1,2}$
}

Deregulation and activation of the phosphoinositide 3-kinase (PI3K)/Akt/mammalian (or mechanistic) target of rapamycin (mTOR) pathway have a major role in proliferation and cell survival in breast cancer. However, as single agents, mTOR inhibitors have had modest antitumor efficacy. In this study, we evaluated the effects of vertical inhibition of mTOR and Akt in breast cancer cell lines and xenografts. We assessed the effects of mTOR inhibitor rapamycin and Akt inhibitor MK-2206, given as single drugs or in combination, on cell signaling, cell proliferation and apoptosis in a panel of cancer cell lines in vitro. The antitumor efficacy was tested in vivo. We demonstrated that MK-2206 inhibited Akt phosphorylation, cell proliferation and apoptosis in a dose-dependent manner in breast cancer cell lines. Rapamycin inhibited S6 phosphorylation and cell proliferation, and resulted in lower levels of apoptosis induction. Furthermore, the combination treatment inhibited phosphorylation of Akt and S6, synergistically inhibited proliferation and induced apoptosis with a higher efficacy. In vivo combination inhibited tumor growth more than either agent alone. Our data suggest that a combination of Akt and mTOR inhibitors have greater antitumor activity in breast cancer cells, which may be a viable approach to treat patients.

Oncogenesis (2017) 6, e385; doi:10.1038/oncsis.2017.86; published online 9 October 2017

\section{INTRODUCTION}

The phosphoinositide 3-kinase (PI3K)/Akt/mammalian (or mechanistic) target of rapamycin (mTOR) pathway regulates cell growth, protein translation, autophagy, metabolism and cell survival. Deregulation and activation of this pathway is associated with tumorigenesis and cancer progression. One or more $\mathrm{PI} 3 \mathrm{~K}$ pathway components were altered in $38 \%$ of cancer patients. ${ }^{1}$ Phosphatase and tensin homolog (PTEN) loss (30\% by immunohistochemistry), PIK3CA (13\%), PTEN (6\%) and AKT1 (1\%) mutations are common and more frequently observed with hormone receptor overexpression (androgen, progesterone or estrogen receptor) or human epidermal growth factor receptor 2 (HER2) amplification. ${ }^{1}$ In vitro data suggest that tumors with a low level PTEN expression or a mutant PIK3CA depend on Akt for oncogenic signaling, such as elevation of phosphorylated Akt levels, is frequently observed in breast cancer and indicate poor prognosis. ${ }^{2}$ Furthermore, in breast cancer, $\mathrm{PI} 3 \mathrm{~K} / \mathrm{Akt} / \mathrm{mTOR}$ pathway is associated with resistance to endocrine therapy, HER2-directed therapy and cytotoxic therapy. ${ }^{3,4}$

Rapamycin is an allosteric inhibitor of mTOR. Everolimus, a rapamycin analog (rapalog), is approved by US Food and Drug Administration in combination with exemestane to treat postmenopausal women with advanced hormone receptor positive, HER2-negative breast cancer. Rapalogs have also been approved for the treatment of neuroendocrine tumors, renal cell carcinoma and subependymal giant cell astrocytoma associated with tuberous sclerosis. Mostly, therapeutic effects were observed in a subset of patients that was leading to disease stabilization rather than tumor regression. The efficacy is likely limited by feedback regulations within the pathway and crosstalk with other pathways. Under normal conditions, feedback regulation from mTOR complex 1 (mTORC1)/S6K1 to insulin receptor substrate-1 (IRS-1) attenuates cell growth signals. ${ }^{5-7}$ In addition, S6K1 phosphorylates rictor and reduces $\mathrm{mTOC} 2$ signaling. ${ }^{8}$ In some cancer cells treated with rapalogs, these two feedback loops can enhance PI3K/Akt signaling. The effect of this Akt activation remains unclear; it is proposed as a marker of resistance but also as an indicator of rapamycin activity, thus a marker of sensitivity. ${ }^{9,10}$ Thus, combination treatments with chemotherapeutic or small molecule inhibitor agents are advised.

MK-2206 (Merck Oncology) is an allosteric inhibitor of Akt. It predominantly inhibits Akt $1 / 2$ in nanomolar range and has reduced potency against Akt3. ${ }^{11}$ It has potent anti-proliferative activity in cell lines with a PIK3CA activating mutation, inactivation of PTEN and amplification or mutation of Akt. ${ }^{11}$ Inhibition of phosphorylation at both Akt T308 and $\mathrm{S} 473$ residues results in inhibition of Akt signaling and cell cycle progression, while an increase in apoptosis. ${ }^{12}$ It has been shown to synergize with cytotoxic agents and pathway inhibitors in vitro and in vivo. ${ }^{13,14}$ MK-2206 monotherapy demonstrated limited antitumor effect in phase II trials and currently it is being tested in combination with chemotherapeutics and small molecule inhibitors. ${ }^{15}$

To increase drug efficacy and overcome resistance, multiple key mediators of tumor survival signaling pathways are being targeted. First approach is to use two inhibitors targeting two pathways (parallel inhibition), such as PI3K/Akt/mTOR and Raf/

\footnotetext{
${ }^{1}$ Department of Surgical Oncology, The University of Texas MD Anderson Cancer Center, Houston, TX, USA; ${ }^{2}$ Department of Investigational Cancer Therapeutics, The University of Texas MD Anderson Cancer Center, Houston, TX, USA and ${ }^{3}$ Department of Biostatistics, The University of Texas MD Anderson Cancer Center, Houston, TX, USA. Correspondence:

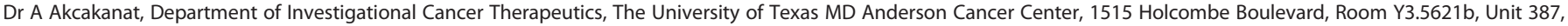
Houston, TX 77030, USA.

E-mail: aakcakanat@mdanderson.org

${ }^{4}$ Current address: Department of Surgery, Korea University College of Medicine, \#80, Guro-dong, Guro-gu, Seoul 152-703, Republic of Korea.

${ }^{5}$ Current address: Department of General Surgery, Chiba University Graduate School of Medicine 1-8-1 Inohana Chuo-ku, Chiba 260-8670, Japan.

${ }^{6}$ Current address: Pfizer Inc. 445 Eastern Point Road, Groton, CT 06340, USA.

Received 24 May 2017; revised 31 July 2017; accepted 24 August 2017
} 
MEK/Erk pathways. ${ }^{16}$ A second method is targeting a pathway at two points (vertical inhibition) by using a dual inhibitor or two inhibitors. There are several dual inhibitors now, and to overcome negative feedback loop Akt activation, the vertical inhibition of PI3K/Akt/mTOR pathway has been studied. ${ }^{17}$ In this study, we evaluated the antitumor growth effect of rapamycin and MK-2206 combination in vitro and in vivo. Our objective was to determine whether this combination would be synergistic. We tested the effect of this combination on cell proliferation, cell cycle progression and apoptosis using breast cancer cell lines.

\section{RESULTS}

MK-2206 inhibits Akt signaling but does not inhibit S6K axis in every cell line

We treated six breast cancer cell lines of different subtypes and genomic backgrounds with MK-2206 at concentration of $500 \mathrm{nM}$ and harvested the cells after 2, 24 and $72 \mathrm{~h}$ (Figure 1a). Akt phosphorylation was inhibited at $2 \mathrm{~h}$ and this inhibition was sustained for at least $72 \mathrm{~h}$. S6K and S6 phosphorylation was decreased in all cell lines. However, a biphasic pattern was observed in in HCC1954, MCF7 and MDA-MB-231, S6K and S6 phosphorylation was initially increased at $2 \mathrm{~h}$ and decreased in late time points. In ZR75-1 cell line, 4E-BP1 phosphorylation was inhibited at $2 \mathrm{~h}$ but in MDA-MB-468 cell line, there was no inhibition. The other cell lines did not have a strong phospho-4EBP1 expression and it was difficult to assess its regulation.

Next, we tested MK-2206 and rapamycin in combination. We selected three rapamycin-sensitive cell lines ${ }^{10}$ and treated with various doses of both drugs for $72 \mathrm{~h}$ (Figure 1b). 56 and Akt were selected as the markers to confirm rapamycin and MK-2206 activity, respectively. High-dose MK-2206 and rapamycin combination resulted in almost complete inhibition of phosphorylation of both $\mathrm{S} 6$ and Akt in all three cell lines. We did not observe a significant decrease in Akt phosphorylation in ZR75-1 cell line with low-dose MK-2206 treatment alone, particularly at Akt T308, and only the combination was effective.

MK-2206 and rapamycin synergistically inhibit breast cancer cell growth

In a panel of six breast cancer cell lines, combination index $(\mathrm{Cl})$ was calculated based on dose-effect levels of median-effect plots of MK-2206 alone, rapamycin alone and their combination at fixed molar ratios. $\mathrm{Cl}$ values were consistently $<1$ for five cell lines indicating synergy (Figure 2a). HCC1954 and MDA-MB-468 cell lines are resistant to MK-2206, ${ }^{12}$ and, at effective dose 90 (ED90) there was more synergy compared with ED50 and ED75. This was

a

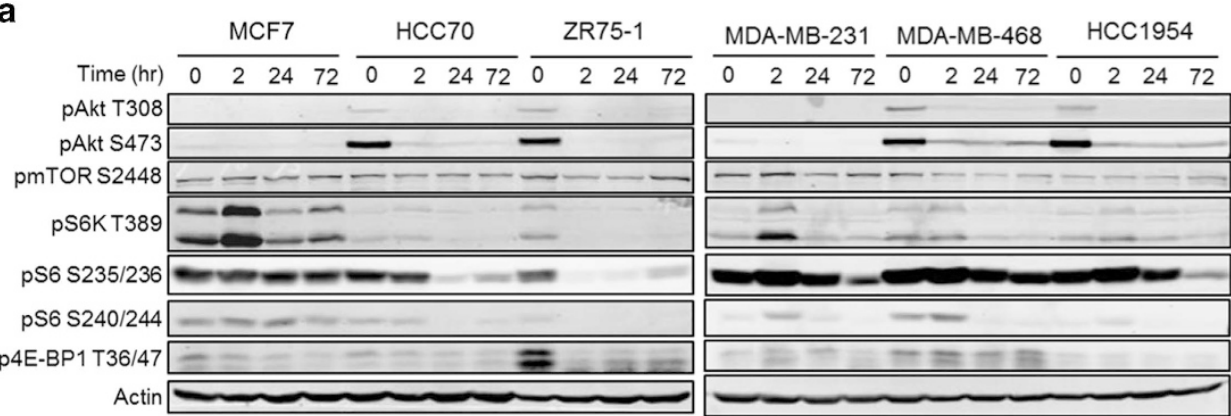

b

MCF7

ZR75-1

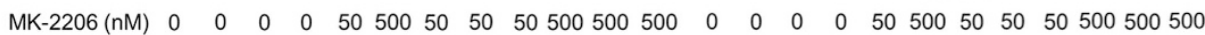
$\begin{array}{llllllllllllllllllllllllll}\text { Rapamycin (nM) } & 0 & 1 & 10 & 100 & 0 & 0 & 1 & 10 & 100 & 1 & 10 & 100 & 0 & 1 & 10 & 100 & 0 & 0 & 1 & 10 & 100 & 1 & 10 & 100\end{array}$

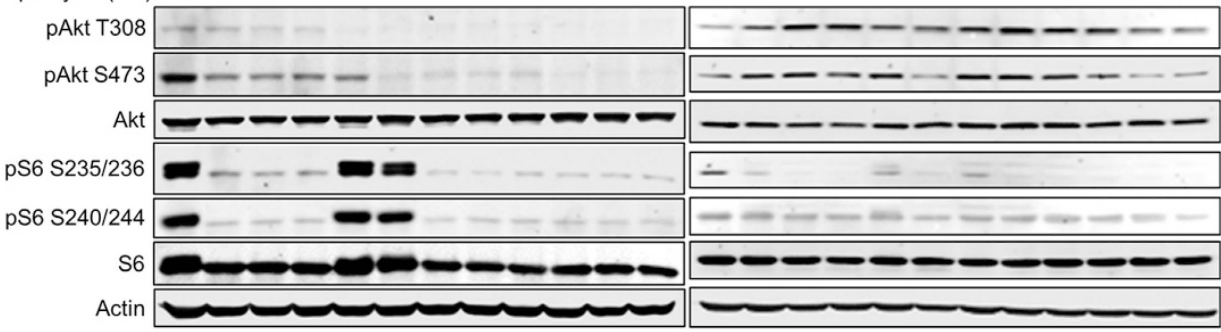

MDA-MB-468

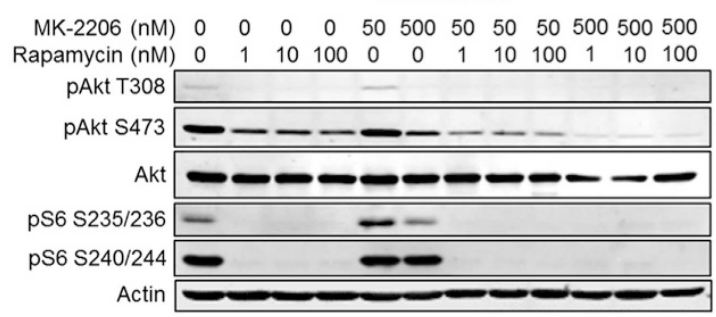

Figure 1. MK-2206 and rapamycin regulate phosphorylation of Akt/mTOR pathway proteins. (a) A panel of breast cancer cell lines was treated with MK-2206 $500 \mathrm{~nm}$ for 2, 24 or $72 \mathrm{~h}$. The changes in phosphorylation status of Akt, mTOR, S6K, S6 and 4E-BP1 in time was analyzed by western blotting using phospho-specific antibodies. (b) Three breast cancer cell lines were treated with various doses of MK-2206, rapamycin and their combination for $72 \mathrm{~h}$. Regulation of Akt and mTOR signaling was demonstrated by western blotting. All experiments were repeated three times. 
a

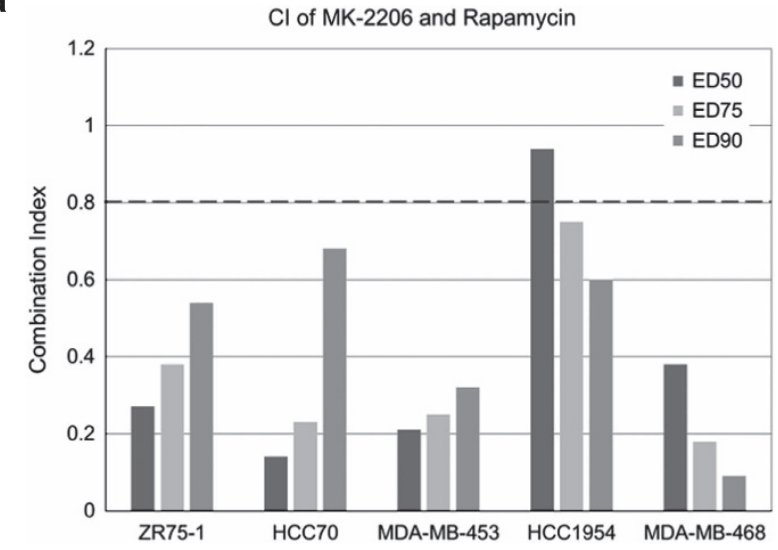

b

b

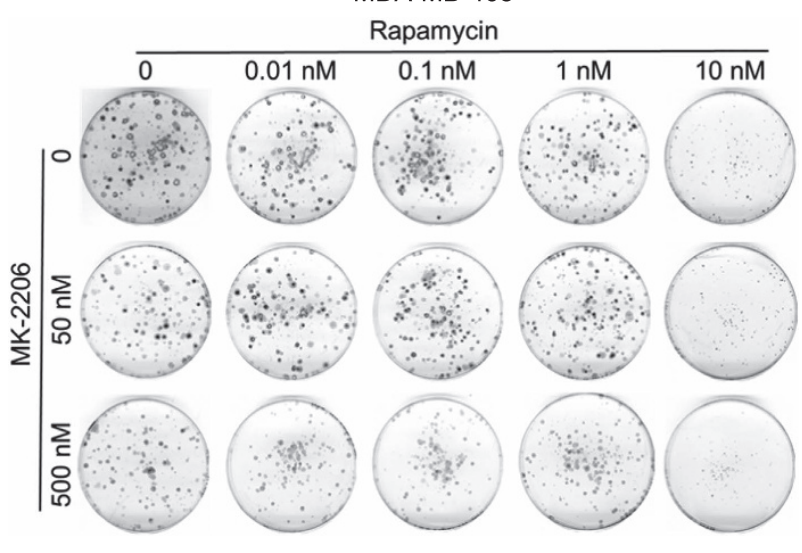

\begin{tabular}{|c|c|c|c|c|}
\hline \multirow{2}{*}{ Cell line } & $\begin{array}{c}\text { MK-2206:rapamycin } \\
\text { molar combination } \\
\text { ratio }\end{array}$ & \multicolumn{3}{|c|}{ Combination Index } \\
\cline { 3 - 5 } & $5: 1$ & ED50 & ED75 & ED90 \\
\hline ZR75-1 & $1: 2$ & 0.27 & 0.38 & 0.54 \\
\hline HCC70 & $5: 1$ & 0.14 & 0.23 & 0.68 \\
\hline MDA-MB-453 & $1: 2$ & 0.21 & 0.25 & 0.32 \\
\hline HCC1954 & $500: 1$ & 0.94 & 0.75 & 0.6 \\
\hline MDA-MB-468 & & 0.38 & 0.18 & 0.09 \\
\hline
\end{tabular}

Figure 2. Combining MK-2206 and rapamycin synergistically suppress growth in vitro. (a) Five breast cancer cell lines were treated with vehicle, MK-2206, rapamycin or a combination of MK-2206 and rapamycin for $96 \mathrm{~h}$ in triplicates. The effect on cell growth was assessed by SRB assay and $\mathrm{Cl}$ values were calculated. The chart displays $\mathrm{Cl}$ of MK-2206 and rapamycin combination at ED50, ED75 and ED90. Cl $<0.8$ represents synergy. (b) MDA-MB-468 cell lines were treated with vehicle, and increasing concentrations of MK-2206, rapamycin or a combination MK-2206 and rapamycin in triplicates. The colonies were fixed and stained. The surface area occupied by colonies is normalized to vehicle control. One of the three experiment in triplicate cultures was shown as mean \pm s.e.m.

a

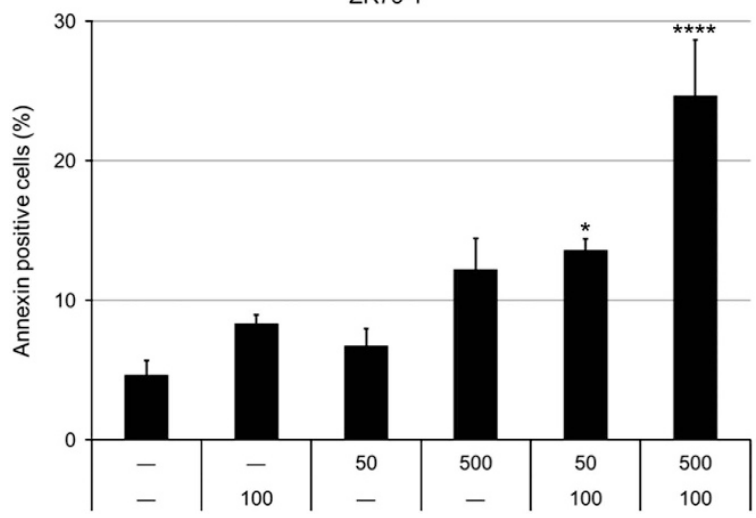

b

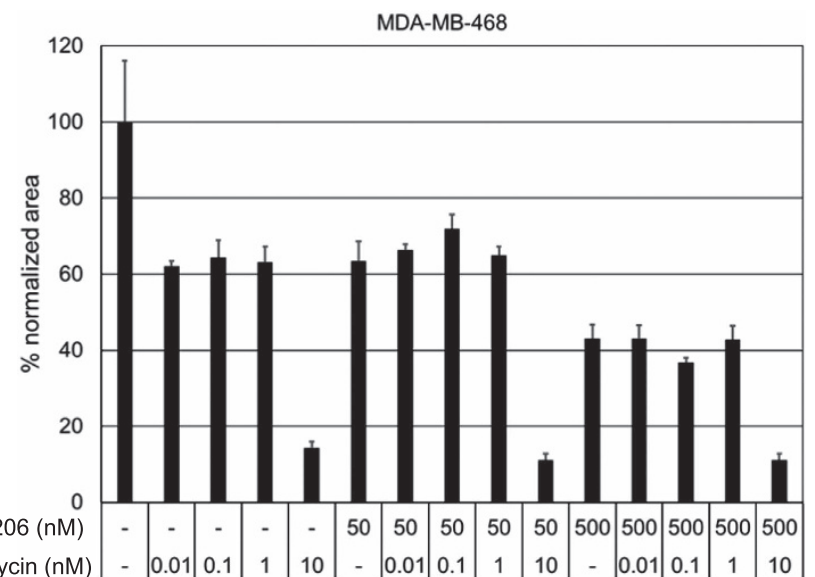

\begin{tabular}{c|c|c|c|c|c|c|c|c|c|c|c|c|c|c|c|} 
MK-2206 (nM) & - & - & - & - & - & 50 & 50 & 50 & 50 & 50 & 500 & 500 & 500 & 500 & 500 \\
Rapamycin (nM) & - & 0.01 & 0.1 & 1 & 10 & - & 0.01 & 0.1 & 1 & 10 & - & 0.01 & 0.1 & 1 & 10
\end{tabular}

Figure 3. Combining MK-2206 and rapamycin synergistically increase apoptosis in vitro. (a) ZR75-1 cell line was treated with vehicle, MK-2206, rapamycin or a combination of MK-2206 and rapamycin for $72 \mathrm{~h}$ in triplicates. The percentages of annexin V-positive cells were determined by flow cytometry and compared. Average of three experiments is represented (mean \pm s.e.m.; ${ }^{*} P<0.05 ;{ }^{* * *} P<0.0001$ ). (b) ZR75-1 cells were treated with vehicle, MK-2206, rapamycin or a combination of MK-2206 and rapamycin for $72 \mathrm{~h}$. Western blotting was conducted to assess Akt phosphorylation and apoptosis. All experiments were repeated three times.

the opposite for the other three sensitive cell lines, there was more synergy at ED50. We were not able to calculate $\mathrm{Cl}$ for MDAMB-231 cell line. As reported previously, this cell line was resistant to both MK-2206 and rapamycin. ${ }^{10,12}$
We performed colony formation assay to measure growth inhibition because of treatment with MK-2206, rapamycin and their combination (Figure 2b). Quantitation and normalization of the colony formation assay was performed to provide and 

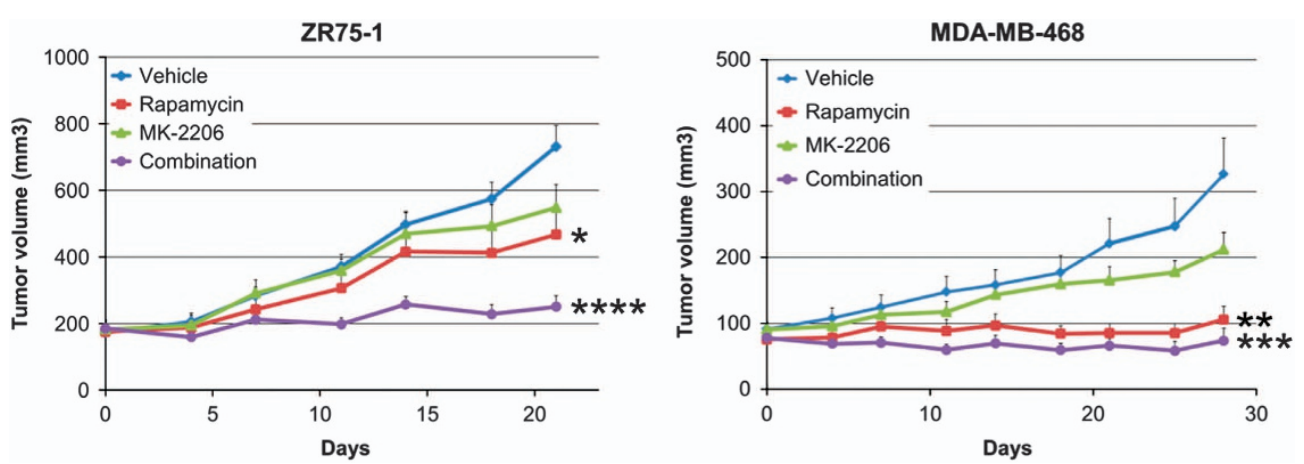

Figure 4. Combining MK-2206 and rapamycin synergistically suppress growth in vivo. Mice bearing ZR75-1 or MDA-MB-468 xenografts were treated with vehicle, MK-2206 $240 \mathrm{mg} / \mathrm{kg}$, rapamycin $10 \mathrm{mg} / \mathrm{kg}$, or a combination of MK-2206 and rapamycin at the same doses once a week. Treatment group tumor volumes were compared with control at the last day of the experiment. Data were represented as mean \pm s.e.m. $\left({ }^{*} P<0.05 ;{ }^{* *} P<0.01 ;{ }^{* *} P<0.001 ;{ }^{* * * * P}<0.0001\right)$.

objective measurement of growth inhibition. Compared with control, all treatment groups significantly inhibited growth $(P$ values ranging from $<0.05$ to $<0.0001)$. However, combinations at various doses did not show a reduction of growth compared with single-agent treatments.

\section{MK-2206 induces apoptosis}

ZR75-1 cell line was treated with vehicle, rapamycin, MK-2206 and their combination for $72 \mathrm{~h}$. The percentages of annexin V-positive cells were determined by flow cytometry (Figure 3a). Both lowand high-dose MK-2206 combined with rapamycin caused a significant increase in apoptotic cell death (at $50 \mathrm{~nm}, P<0.05$, at $500 \mathrm{~nm}, P<0.0001)$. Compared with high-dose MK-2206 alone, high-dose MK-2206 combination with rapamycin doubled the percentage of apoptotic cells (mean \pm s.e.m.: MK-2206, 12.2 \pm 2.0 ; combination, 24.6 $\pm 4.0 ; P<0.001$ ). Western blotting of the PARP cleavage in response to MK-2206, rapamycin and combination treatment for $72 \mathrm{~h}$ is shown in Figure 3b. PARP cleavage clearly increased with high-dose MK-2206 treatment. The combination with rapamycin enhanced the PARP cleavage observed with lowdose MK-2206. Under the same treatment conditions, we did not observe apoptosis in MDA-MB-468 cell line (data not shown).

Combination of MK-2206 and rapamycin inhibits tumor growth in vivo

Tumor volumes of four groups were compared at the final day of treatment (Figure 4). In both ZR75-1 and MDA-MB-468 cell line xenografts, MK-2206 alone inhibited tumor growth; however, this inhibition did not reach significance level of 0.05 . Rapamycin alone was more effective compared with MK-2206 (vehicle vs rapamycin, ZR75-1 $P<0.05$ and MDA-MB-468 $P<0.01$ ) and the greatest inhibition of tumor growth was delivered by the MK-2206 and rapamycin combination treatment (vehicle vs combination, ZR75$1 P<0.0001$ and MDA-MB-468 $P<0.001)$.

Functional proteomics demonstrated inhibition of Akt/mTOR pathway

The reverse phase proteomics array (RPPA) core facility antibody panel is enriched in PI3K/Akt/mTOR pathway proteins. MDAMB-468 cell line has PTEN loss, ${ }^{18}$ high EGFR expression ${ }^{19}$ and activated PI3K/Akt/mTOR pathway. ZR75-1 has a PTEN (L108R) mutation ${ }^{20}$ with very weak PTEN expression and high levels of pAkt. ${ }^{18}$ Regardless of both cell lines having Akt/mTOR pathway activation, they showed alteration of different markers (Supplementary Figure 1). The combination treatment arm showed that pAkt, its downstream target pGSK 3, and mTOR downstream target p4E-BP1 were inhibited. Notch 1 and tuberin were downregulated, and $\mathrm{XXL}$ and collagen VI were upregulated in both cell line xenografts.

\section{DISCUSSION}

Activation of $\mathrm{PI} 3 \mathrm{~K} / \mathrm{Akt} / \mathrm{mTOR}$ pathway is a central event in many types of cancer and represents a promising target for new treatment strategies. However, there is modest antitumor activity of single-agent therapies suggesting that there is a need for drug combinations to induce superior clinical responses. In this study, we investigated the vertical targeting of PI3K/Akt/mTOR pathway in breast cancer cells. After MK-2206 and rapamycin treatment, key downstream proteins within Akt/mTOR pathway were dephosphorylated in breast cancer cell lines, including Akt, mTOR, S6 and $4 \mathrm{E}-\mathrm{BP} 1$. This combination produced a synergistic effect against breast cancer cell proliferation in vitro. Furthermore, this combination induced apoptosis in vitro and inhibited tumor growth more compared with single drug groups in animal models. Our drug dosing schedule in mice was similar to the schedule in humans and we did not observe any signs of toxicity. The data support dual targeting of PI3K/Akt/mTOR pathway in cancer treatment.

Western blotting showed that rapamycin and MK-2206 combination blocked Akt/mTOR signaling completely. However, MK-2206 as single drug activated S6K and S6 at 2-h time point. Biphasic phosphorylation of $\mathrm{S} 6$ was also reported after rapamycin treatment. ${ }^{21}$ To our knowledge, there is no clear explanation of this event and this finding indicates complexity of S6K/S6 phosphorylation.

In many studies, pS6 is used as a marker of mTOR activity, however, in our study RPPA did not show a decrease in pS6 levels. We collected xenograft tumors $24 \mathrm{~h}$ later following the last injection of drugs. It is possible that this period was too long to detect alterations in phosphorylation status of target proteins. Interestingly, combination treatment regulated expression levels of four proteins in both cell line xenografts. Of the two upregulated proteins, collagen VI expression was reported to be upregulated in breast cancer promoting tumor progression and metastasis. $^{22} \mathrm{AXL}$ overexpression is poor prognostic and contributes to the functional skewing of macrophage functions in triple-negative breast cancer (TNBC). ${ }^{23}$ Vimentin-positive and AXL high-expressing TNBCs have shorter recurrence-free and overall survival. ${ }^{24}$ Of the two downregulated proteins, in a meta-analysis, Notch 1 is found to be overexpressed in basal subtype of breast cancer and associated with transition from ductal carcinoma in situ to invasive cancer. ${ }^{25}$ Tuberin expression is low in breast cancer tumors compared with normal tissues, and patients with recurrence and mortality have been reported to have significantly lower levels compared with those who remained disease free. ${ }^{26}$ 
Interestingly, three of four regulated proteins are associated with poor prognosis. We need to validate these results in a larger number of preclinical models to understand the underlying mechanisms. These alterations may represent adaptive responses to facilitate survival upon treatment with PI3K/Akt/mTOR inhibitors. This opens up the possibility that targeting these survival pathways in combination, may further enhance antitumor efficacy.

Elevated phospho-Akt levels predicted increased sensitivity to mTOR inhibition in vitro. ${ }^{10}$ In a rapamycin-resistant diffuse large B-cell lymphoma model, a gene expression profile was generated. ${ }^{27}$ This model identified lymphoma subsets that were resistant to mTOR inhibitor therapy and mapped compounds targeting Akt as a way of reversing mTOR inhibitor resistance. ${ }^{27}$ MK-2206 and a rapalog combination was synergistic in other types of malignancies such as B-precursor acute lymphoblastic leukemia, ${ }^{28}$ cholangiocarcinoma, ${ }^{29}$ hepatocellular carcinoma ${ }^{30,31}$ and neuroblastoma ${ }^{32}$ in vitro and in vivo, in multiple myeloma, ${ }^{33}$ gastric cancer ${ }^{34}$ and thyroid cancer ${ }^{35}$ in vitro. Using a genetically engineered mouse model of castration-resistant prostate cancer model, Floc'h et $a l^{36}$ tested combination of MK-2206 and a rapalog ridaforolimus (MK-8669). They reported that the feedback loop activation was observed in human prostate cancer cell lines but not in vivo model, and suggested that different factors, such as mTORC2 activation of Akt, might contribute to the enhanced efficacy. ${ }^{36}$ In an MK-2206-induced acquired resistance model of neuroblastoma cell lines, MK-2206-resistant cells showed increased sensitivity to mTOR (AZD8805) and PDK1 (GSK2334470) inhibitors. ${ }^{37}$ These cells were characterized by low Akt phosphorylation and elevated PDK1/mTOR/S6K activity, and inhibition of PDK1/mTOR/S6K signaling was suggested as a way to overcome the MK-2206 resistance. Recently, Mi et $a l^{38}$ stated that sustained $4 \mathrm{E}-\mathrm{BP} 1$ phosphorylation resulted in rapamycin resistance and effective inhibition of PRAS40 phosphorylation was required to overcome this resistance. Dephosphorylation of PRAS40 represses Akt/mTORC1 regulated 4E-BP1 phosphorylation. To confirm this statement, we selected ZR75-1 cell line, where baseline 4E-BP1 phosphorylation was the highest (Figure 1a) and tested three phosphorylation sites, T36/47, S65 and T70. A significant decrease in PRAS40 phosphorylation was not observed (data not shown). However, combination treatment was more effective in suppressing 4E-BP1 phosphorylation, suggesting that combination therapy may more effectively inhibit cap-dependent translation.

The need for combining MK-2206 with other targeting compounds to treat breast cancer is emphasized in two recently reported studies. In a patient-derived xenograft model of basallike breast cancer, there was synergistic effect of MK-2206 and ridaforolimus on tumor growth and cell proliferation. ${ }^{39}$ PTEN knockdown increased this synergy suggesting a rationale to use this combination in basal-like breast cancer with PTEN loss. A phase I study of MK-2206 and ridaforolimus in advanced cancers was recently reported. The cohort was enriched in breast cancer patients with low RAS gene signature (a score derived from expression of 147 transcripts) or ER+ patients with high Ki-67 index (high proliferation). ${ }^{40}$ Partial and complete response were observed in $12.5 \%$ and $14.3 \%$ of the patients, respectively. The combination had signal of activity in heavily treated breast cancer patients with exhibiting PI3K pathway dependence. Precinically, other Akt inhibitors were also tested in combination with rapalogs. In human breast cancer cell lines, rapamycin and A-443654 combination induced G2-M arrest and apoptosis. ${ }^{41}$ Use of perifosine and temsirolimus to treat mice with gliomas resulted in decreased Akt and mTOR signaling, decreased proliferation and increased cell death. ${ }^{42}$ All listed preclinical studies taken together demonstrate that inhibition of multiple components of Akt/mTOR pathway is superior to inhibition of a single target.

The advantage of dual targeting of mitogen-activated protein kinase (MAPK) pathway has been clearly demonstrated in melanoma. MEK inhibitors significantly enhanced antitumor efficacy in when given in combination with BRAF inhibitors for BRAF V600E mutant melanoma both in preclinical models and in clinical trials, leading to the FDA approval of dabrafenib and trametinib, ${ }^{43-45}$ as well as vemurafenib with cobimetinib. ${ }^{46}$ Further, dual pathway inhibition decreased the toxicity profile, especially by decreasing development of cutaneous malignancies. ${ }^{45}$ In contrast, dual inhibition of PI3K and mTOR with dual inhibitors has been more challenging because of the toxicity profile of these agents. Similarly, Akt inhibitors and mTOR inhibitors would be expected to have overlapping toxicity. Indeed, in phase I the combination of ridaforolimus and MK-2206 was considered tolerable but several adverse events were common, such as rash (44\%), stomatitis (39\%), diarrhea (28\%) and decreased appetite $(28 \%){ }^{40}$ Thus, further work is needed to identify optimal agents, dose and schedule for vertical inhibition of PI3K/Akt/mTOR signaling with the combination of Akt inhibitor and mTOR inhibitor. There are three more ongoing phase I trials giving a combination of $\mathrm{PI} 3 \mathrm{~K}$ and $\mathrm{mTOR}$ inhibitors, and there are no results yet. $^{47-49}$

In summary, considering that a major part of human breast cancers have activation of PI3K/Akt/mTOR pathway, targeting Akt and mTOR together in the treatment of this disease may enhance antitumor efficacy. Further studies are needed to determine the role of this therapeutic approach in different molecular subtypes and the clinical feasibility of this approach.

\section{MATERIALS AND METHODS}

Cell lines, cultures and reagents

HCC70, HCC1954, MCF7, MDA-MB-231, MDA-MB-453, MDA-MB-468 and ZR75-1 breast cancer cell lines were obtained from American Tissue Culture Collection (Manassas, VA, USA). All cell lines were cultured in Dulbecco's modified Eagle's medium/F12 supplemented with $10 \%$ fetal bovine serum at $37^{\circ} \mathrm{C}$ and humidified in $5 \% \mathrm{CO}_{2}$.

Captisol, dimethyl sulphoxide (DMSO), MK-2206 and rapamycin were purchased from CYDEX Pharmaceuticals (Lenexa, KS, USA), Sigma Chemical Company (St Louis, MO, USA), Selleckchem (Houston, TX, USA) and LC Laboratories (Woburn, MA, USA), respectively. For in vitro experiments, stock solutions of rapamycin and MK-2206 were prepared in DMSO at $10 \mathrm{~mm}$. For in vivo experiments, stock solution of rapamycin was prepared in DMSO at $10 \mathrm{mg} / \mathrm{ml}$, whereas MK-2206 was formulated in a $30 \%(\mathrm{w} / \mathrm{v})$ Captisol solution.

\section{Cell proliferation assay}

Cells were plated at 1500-2000 cells per well in 96-well plates and allowed to attach overnight. Cells were treated with increasing doses of MK-2206, rapamycin or a combination of MK-2206 and rapamycin for 4 days. Cell proliferation was assessed by sulforhodamine B (SRB) assay. ${ }^{50}$ The median inhibitory concentration (IC50) and $\mathrm{Cl}$ were determined from doseresponse curves for 4 days treatment by CalcuSyn (Biosoft, UK). Cl values are presented at ED50, ED75 and ED90, to inhibit growth at 50\%,75\% and $90 \%$, respectively. $\mathrm{Cl}$ indicates synergy $(<0.8)$, additive $(0.8-1.2)$, and antagonism (>1.2).

\section{Colony formation assay}

MDA-MB-468 cells were plated at 2000 cells per plate in $6-\mathrm{cm}$ plates and allowed to attach overnight. Cells were treated with increasing doses of MK-2206, rapamycin or a combination of MK-2206 and rapamycin once a week. Colony formation was assessed by crystal violet staining. Plates were scanned and colonies were counted using the ImageJ software. ${ }^{51}$ Relative colony area was determined by normalizing colony area of each plate to the average colony area of the control.

\section{Annexin $\mathrm{V}$ assay}

Cells were treated for $72 \mathrm{~h}$ and apoptosis was identified by Annexin V Apoptosis kit (Roche, Indianapolis, IN, USA) according to the manufacturer's protocol in the MD Anderson Flow Cytometry and Cellular Imaging Core Facility. Labeled cells were analyzed by flow cytometry and FlowJo (FlowJo, LLC, Ashland, OR, USA). 


\section{Western blotting}

Cultured cells were washed in cold PBS and lysed in buffer containing $62.5 \mathrm{~mm}$ Tris- $\mathrm{HCl}, \mathrm{pH} 6.8,2 \%$ sodium dodecyl sulfate and $25 \%$ glycerol. Protein lysates were separated by sodium dodecyl sulfate-polyacrylamide gel electrophoresis and transferred to $0.2 \mu \mathrm{m}$ nitrocellulose membrane. After blocking with $0.1 \%$ casein, membranes were probed with antibodies to p4E-BP1 T36/47 (\#9459), Akt (\#9272), pAkt T308 (\#13038), pAkt S473 (\#9271), pmTOR S2448 (\#2983), PARP (\#9542), S6 (\#2217), pS6 S235/236 (\#4858), pS6 S240/244 (\#2215), pS6K T389 (\#9234) (Cell Signaling Technologies, Boston, MA, USA) and actin (\#A5441) (Sigma-Aldrich Co., LLC, St Louis, MO, USA). The signals were visualized by Odyssey infrared imaging system (Li-Cor Biosciences, Lincoln, NE, USA).

\section{Reverse phase proteomics array}

RPPA was done in the MD Anderson Cancer Center Functional Proteomics RPPA Core Facility as described previously. ${ }^{10}$ Proteomic profiles of MK-2206, rapamycin and combination treatment xenografts were compared with the vehicle. Relative expression values of proteins were in log2. The median polish normalized RPPA data set consisted of 285 proteins.

\section{In vivo studies}

All animal experiments were approved by the MD Anderson Animal Care and Use Committee following Public Health Service Animal Welfare Assurance \#A334301. ZR75-1 $\left(1 \times 10^{7}\right)$ and MDA-MB-468 $\left(5 \times 10^{6}\right)$ cells were inoculated in the mammary fat pads of female nu/nu mice (Department of Experimental Radiology, MD Anderson). Mice were randomized into combination vehicle control, MK-2206 $240 \mathrm{mg} / \mathrm{kg}$, rapamycin $10 \mathrm{mg} / \mathrm{kg}$, and MK-2206 and rapamycin combination therapy groups. MK-2206 and rapamycin were administered by oral gavage and intraperitoneal injection, respectively. There were seven mice in each group and all treatments were given weekly. Tumor volumes were calculated as previously described. ${ }^{52}$ Mice were killed $24 \mathrm{~h}$ after the last treatment. Tumors were snap-frozen for further analysis.

\section{Statistical analysis}

For in vitro and in vivo studies, comparisons of all groups were carried out by one-way analysis of variance (ANOVA) with Tukey's test for multiple comparisons using (GraphPad Prism software, Inc., La Jolla, CA, USA). Xenograft tumor volumes were compared at the final day of treatment. Data were presented as means \pm s.e. A two-sided $P$-value $<0.05$ was considered significant.

For each cell line xenograft, one-way ANOVA was used to assess the differences in protein expression levels between treatment groups on a marker-by-marker basis. An unadjusted overall F-test $P$-value of $<0.05$ and an unadjusted pairwise comparison $P$-value of $<0.05$ were used to determine if there is any significant difference among any of the means by treatment group. The contrasts of the desired comparisons between treatment groups were performed using the 'multcomp' $\mathrm{R}$ package (https://cran.r-project.org/web/packages/multcomp). To account for multiple testing, the false discovery rates of the overall F-test of the model was estimated using the Benjamini-Hochberg method. $^{53}$

\section{CONFLICT OF INTEREST}

The authors declare no conflict of interest.

\section{ACKNOWLEDGEMENTS}

This work was supported by the NCATS grant UL1 TR000371 (National Center for Advancing Translational Sciences), the Nellie B Connally Breast Cancer Research Endowment, Susan G Komen Foundation for the Cure grant SAC10006 (FM-B), Stand Up to Cancer, and the $\mathrm{NCl}$ Cancer Center Support grant (P30 CA016672). MD Anderson Flow Cytometry and Cellular Imaging Core Facility, and RPPA Core Facility are supported by NCl Cancer Center Support Grant P30 CA16672.

\section{PUBLISHER'S NOTE}

Springer Nature remains neutral with regard to jurisdictional claims in published maps and institutional affiliations.

\section{REFERENCES}

1 Millis SZ, Ikeda S, Reddy S, Gatalica Z, Kurzrock R. Landscape of phosphatidylinositol-3-kinase pathway alterations across 19784 diverse solid tumors. JAMA Oncol 2016; 2: 1565-1573.

2 Saal LH, Johansson P, Holm K, Gruvberger-Saal SK, She QB, Maurer M et al. Poor prognosis in carcinoma is associated with a gene expression signature of aberrant PTEN tumor suppressor pathway activity. Proc Natl Acad Sci USA 2007; 104: 7564-7569.

3 Nahta R. Pharmacological strategies to overcome HER2 cross-talk and trastuzumab resistance. Curr Med Chem 2012; 19: 1065-1075.

4 Paplomata E, O'Regan R. New and emerging treatments for estrogen receptorpositive breast cancer: focus on everolimus. Ther Clin Risk Manage 2013; 9: 27-36.

5 Shi Y, Yan H, Frost P, Gera J, Lichtenstein A. Mammalian target of rapamycin inhibitors activate the AKT kinase in multiple myeloma cells by up-regulating the insulin-like growth factor receptor/insulin receptor substrate-1/phosphatidylinositol 3-kinase cascade. Mol Cancer Ther 2005; 4: 1533-1540.

6 Sun SY, Rosenberg LM, Wang X, Zhou Z, Yue P, Fu H et al. Activation of Akt and elF4E survival pathways by rapamycin-mediated mammalian target of rapamycin inhibition. Cancer Res 2005; 65: 7052-7058.

7 O'Reilly KE, Rojo F, She QB, Solit D, Mills GB, Smith D et al. mTOR inhibition induces upstream receptor tyrosine kinase signaling and activates Akt. Cancer Res 2006; 66: 1500-1508.

8 Hresko RC, Mueckler M. mTOR.RICTOR is the Ser473 kinase for Akt/protein kinase B in 3T3-L1 adipocytes. J Biol Chem 2005; 280: 40406-40416.

9 Wang X, Yue P, Kim YA, Fu H, Khuri FR, Sun SY. Enhancing mammalian target of rapamycin (mTOR)-targeted cancer therapy by preventing mTOR/raptor inhibition-initiated, mTOR/rictor-independent Akt activation. Cancer Res 2008; 68: 7409-7418.

10 Meric-Bernstam F, Akcakanat A, Chen H, Do KA, Sangai T, Adkins F et al. PIK3CA/ PTEN mutations and Akt activation as markers of sensitivity to allosteric mTOR inhibitors. Clin Cancer Res 2012; 18: 1777-1789.

11 Yan L. Abstract \#DDT01-1: MK-2206: A potent oral allosteric AKT inhibitor. AACR Meeting Abstracts. 2009;2009(2_Annual_Meeting):DDT01-1-.

12 Sangai T, Akcakanat A, Chen H, Tarco E, Wu Y, Do KA et al. Biomarkers of response to Akt inhibitor MK-2206 in breast cancer. Clin Cancer Res 2012; 18: 5816-5828.

13 Ewald F, Norz D, Grottke A, Hofmann BT, Nashan B, Jucker M. Dual inhibition of PI3K-AKT-mTOR- and RAF-MEK-ERK-signaling is synergistic in cholangiocarcinoma and reverses acquired resistance to MEK-inhibitors. Invest New Drugs 2014; 32: 1144-1154.

14 Simioni C, Martelli AM, Cani A, Cetin-Atalay R, McCubrey JA, Capitani S et al. The AKT inhibitor MK-2206 is cytotoxic in hepatocarcinoma cells displaying hyperphosphorylated AKT-1 and synergizes with conventional chemotherapy. Oncotarget 2013; 4: 1496-1506.

15 Brown JS, Banerji U. Maximising the potential of AKT inhibitors as anti-cancer treatments. Pharmacol Ther 2017; 172: 101-115.

16 Banerji U, Smith AD, Zivi A, Lorente D, Rihawi K, Tunariu N et al. Dual targeting of RAF-MEK-ERK and PI3K-AKT-mTOR pathways in RAS-mutant cancers: preclinical insights and institutional experience from a clinical trial of binimetinib (MEK162) plus BYL719. J Clin Oncol 2014; 32(15_suppl): e13559.

17 Hassan B, Akcakanat A, Holder AM, Meric-Bernstam F. Targeting the PI3-kinase/ Akt/mTOR signaling pathway. Surg Oncol Clin N Am 2013; 22: 641-664.

18 Weng LP, Smith WM, Dahia PL, Ziebold U, Gil E, Lees JA et al. PTEN suppresses breast cancer cell growth by phosphatase activity-dependent $\mathrm{G} 1$ arrest followed by cell death. Cancer Res 1999; 59: 5808-5814.

19 Hyatt DC, Ceresa BP. Cellular localization of the activated EGFR determines its effect on cell growth in MDA-MB-468 cells. Exp Cell Res 2008; 314: 3415-3425.

20 Forbes SA, Beare D, Gunasekaran P, Leung K, Bindal N, Boutselakis H et al. COSMIC: exploring the world's knowledge of somatic mutations in human cancer. Nucleic Acids Res 2015; 43(Database issue): D805-D811.

21 Chen L, Hu L, Dong JY, Ye Q, Hua N, Wong M et al. Rapamycin has paradoxical effects on S6 phosphorylation in rats with and without seizures. Epilepsia 2012; 53: 2026-2033.

22 Karousou E, D'Angelo ML, Kouvidi K, Vigetti D, Viola M, Nikitovic D et al. Collagen $\mathrm{VI}$ and hyaluronan: the common role in breast cancer. BioMed Res Int 014; 2014: 606458.

23 Bottai G, Raschioni C, Székely B, Di Tommaso L, Szász AM, Losurdo A et al. AXLassociated tumor inflammation as a poor prognostic signature in chemotherapytreated triple-negative breast cancer patients. NPJ Breast Cancer 2016; 2: 16033.

24 Tanaka K, Tokunaga E, Inoue Y, Yamashita N, Saeki H, Okano S et al. Impact of expression of vimentin and Axl in breast cancer. Clin Breast Cancer 2016; 16: 520-6 e2.

25 Yuan X, Zhang M, Wu H, Xu H, Han N, Chu Q et al. Expression of Notch1 correlates with breast cancer progression and prognosis. PLOS ONE 2015; 10: e0131689.

26 Jiang WG, Sampson J, Martin TA, Lee-Jones L, Watkins G, Douglas-Jones A et al. Tuberin and hamartin are aberrantly expressed and linked to clinical outcome in 
human breast cancer: the role of promoter methylation of TSC genes. Eur J Cancer 2005; 41: 1628-1636.

27 Petrich AM, Leshchenko V, Kuo PY, Xia B, Thirukonda VK, Ulahannan N et al. Akt inhibitors MK-2206 and nelfinavir overcome mTOR inhibitor resistance in diffuse large B-cell lymphoma. Clin Cancer Res 2012; 18: 2534-2544.

28 Neri LM, Cani A, Martelli AM, Simioni C, Junghanss C, Tabellini G et al. Targeting the PI3K/Akt/mTOR signaling pathway in B-precursor acute lymphoblastic leukemia and its therapeutic potential. Leukemia 2014; 28: 739-748.

29 Ewald F, Grabinski N, Grottke A, Windhorst S, Norz D, Carstensen L et al. Combined targeting of AKT and mTOR using MK-2206 and RAD001 is synergistic in the treatment of cholangiocarcinoma. Int J Cancer 2013; 133: 2065-2076.

30 Grabinski N, Ewald F, Hofmann BT, Staufer K, Schumacher U, Nashan B et al. Combined targeting of AKT and mTOR synergistically inhibits proliferation of hepatocellular carcinoma cells. Mol Cancer 2012; 11: 85.

31 Ewald F, Norz D, Grottke A, Bach J, Herzberger C, Hofmann BT et al. Vertical targeting of AKT and mTOR as well as dual targeting of AKT and MEK signaling is synergistic in hepatocellular carcinoma. J Cancer 2015; 6: 1195-1205.

32 Li Z, Yan S, Attayan N, Ramalingam S, Thiele CJ. Combination of an allosteric Akt Inhibitor MK-2206 with etoposide or rapamycin enhances the antitumor growth effect in neuroblastoma. Clin Cancer Res 2012; 18: 3603-3615.

33 Ramakrishnan V, Kimlinger T, Haug J, Painuly U, Wellik L, Halling T et al. Antimyeloma activity of Akt inhibition is linked to the activation status of PI3K/Akt and MEK/ERK pathway. PLoS ONE 2012; 7: e50005.

34 Ji D, Zhang Z, Cheng L, Chang J, Wang S, Zheng B et al. The combination of RAD001 and MK-2206 exerts synergistic cytotoxic effects against PTEN mutant gastric cancer cells: involvement of MAPK-dependent autophagic, but not apoptotic cell death pathway. PLoS ONE 2014; 9: e85116.

35 Liu R, Liu D, Trink E, Bojdani E, Ning G, Xing M. The Akt-specific inhibitor MK2206 selectively inhibits thyroid cancer cells harboring mutations that can activate the PI3K/Akt pathway. J Clin Endocrinol Metab 2011; 96: E577-E585.

36 Floc'h N, Kinkade CW, Kobayashi T, Aytes A, Lefebvre C, Mitrofanova A et al. Dual targeting of the Akt/mTOR signaling pathway inhibits castration-resistant prostate cancer in a genetically engineered mouse model. Cancer Res 2012; 72: 4483-4493.

37 Qi L, Toyoda H, Xu DQ, Zhou Y, Sakurai N, Amano K et al. PDK1-mTOR signaling pathway inhibitors reduce cell proliferation in MK2206 resistant neuroblastoma cells. Cancer Cell Int 2015; 15: 91.

$38 \mathrm{Mi}$ W, Ye Q, Liu S, She QB. AKT inhibition overcomes rapamycin resistance by enhancing the repressive function of PRAS40 on mTORC1/4E-BP1 axis. Oncotarget 2015; 6: 13962-13977.

39 Xu S, Li S, Guo Z, Luo J, Ellis MJ, Ma CX. Combined targeting of mTOR and AKT is an effective strategy for basal-like breast cancer in patient-derived xenograft models. Mol Cancer Ther 2013; 12: 1665-1675.

40 Gupta S, Argiles G, Munster PN, Hollebecque A, Dajani O, Cheng JD et al. A phase I trial of combined ridaforolimus and MK-2206 in patients with advanced malignancies. Clin Cancer Res 2015; 21: 5235-5244.

41 Zheng J, Hudder A, Zukowski K, Novak RF. Rapamycin sensitizes Akt inhibition in malignant human breast epithelial cells. Cancer Lett 2010; 296: 74-87.
42 Pitter KL, Galban CJ, Galban S, Tehrani OS, Li F, Charles N et al. Perifosine and CCI 779 co-operate to induce cell death and decrease proliferation in PTEN-intact and PTEN-deficient PDGF-driven murine glioblastoma. PLOS ONE 2011; 6: e14545.

43 Greger JG, Eastman SD, Zhang V, Bleam MR, Hughes AM, Smitheman KN et al. Combinations of BRAF, MEK, and PI3K/mTOR inhibitors overcome acquired resistance to the BRAF inhibitor GSK2118436 dabrafenib, mediated by NRAS or MEK mutations. Mol Cancer Ther 2012; 11: 909-920.

44 Administration USFaD FDA Approval for Dabrafenib. Retrieved from https://www. cancer.gov/about-cancer/treatment/drugs/fda-dabrafenib.

45 Long GV, Stroyakovskiy D, Gogas H, Levchenko E, de Braud F, Larkin J et al. Dabrafenib and trametinib versus dabrafenib and placebo for Val600 BRAFmutant melanoma: a multicentre, double-blind, phase 3 randomised controlled trial. Lancet 2015; 386: 444-451.

46 Larkin J, Ascierto PA, Dreno B, Atkinson V, Liszkay G, Maio M et al. Combined vemurafenib and cobimetinib in BRAF-mutated melanoma. N Engl J Med 2014; 371: 1867-1876.

47 A Phase I Study of BKM120 and Everolimus in Advanced Solid Malignancies 2017, Retrieved from https://clinicaltrials.gov/ (Identification no. NCT01470209).

48 Study of Safety and Efficacy of Alpelisib With Everolimus or Alpelisib With Everolimus and Exemestane in Advanced Breast Cancer Patients, Renal Cell Cancer and Pancreatic Tumors. 2017. Retrieved from https://clinicaltrials.gov/ (Identification no. NCT02077933).

49 Phase 1b Study of MLN0128 in Combination With MLN1117 in Adult Patients With Advanced Nonhematologic Malignancies. 2017. Retrieved from https://clin icaltrials.gov/ (Identification no. NCT01899053).

50 Moreno A, Akcakanat A, Munsell MF, Soni A, Yao JC, Meric-Bernstam F. Antitumor activity of rapamycin and octreotide as single agents or in combination in neuroendocrine tumors. Endocr-Relat Cancer 2008; 15: 257-266.

51 Schneider CA, Rasband WS, Eliceiri KW. NIH Image to ImageJ: 25 years of image analysis. Nat Methods 2012; 9: 671-675.

52 Mondesire WH, Jian W, Zhang H, Ensor J, Hung MC, Mills GB et al. Targeting mammalian target of rapamycin synergistically enhances chemotherapy-induced cytotoxicity in breast cancer cells. Clin Cancer Res 2004; 10: 7031-7042.

53 Benjamini $Y$, Hochberg $Y$. Controlling the false discovery rate: a practical and powerful approach to multiple testing. J R Stat Soc Series B (Methodological) 1995; 57: $289-300$.

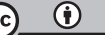

Oncogenesis is an open-access journal published by Nature Publishing Group. This work is licensed under a Creative Commons Attribution 4.0 International License. The images or other third party material in this article are included in the article's Creative Commons license, unless indicated otherwise in the credit line; if the material is not included under the Creative Commons license, users will need to obtain permission from the license holder to reproduce the material. To view a copy of this license, visit http://creativecommons.org/licenses/by/4.0/

(c) The Author(s) 2017

Supplementary Information accompanies this paper on the Oncogenesis website (http://www.nature.com/oncsis) 